\title{
Equilibria with coordination failures
}

Citation for published version (APA):

Herings, P. J. J., Laan van der, G., \& Talman, A. J. J. (2009). Equilibria with coordination failures. Journal of Mathematical Economics, 45, 23-37. https://doi.org/10.1016/j.jmateco.2007.11.002

Document status and date:

Published: 01/01/2009

DOI:

10.1016/j.jmateco.2007.11.002

Document Version:

Publisher's PDF, also known as Version of record

Document license:
Taverne

\section{Please check the document version of this publication:}

- A submitted manuscript is the version of the article upon submission and before peer-review. There can be important differences between the submitted version and the official published version of record.

People interested in the research are advised to contact the author for the final version of the publication, or visit the DOI to the publisher's website.

- The final author version and the galley proof are versions of the publication after peer review.

- The final published version features the final layout of the paper including the volume, issue and page numbers.

Link to publication

\footnotetext{
General rights rights.

- You may freely distribute the URL identifying the publication in the public portal. please follow below link for the End User Agreement:

www.umlib.nl/taverne-license

Take down policy

If you believe that this document breaches copyright please contact us at:

repository@maastrichtuniversity.nl

providing details and we will investigate your claim.
}

Copyright and moral rights for the publications made accessible in the public portal are retained by the authors and/or other copyright owners and it is a condition of accessing publications that users recognise and abide by the legal requirements associated with these

- Users may download and print one copy of any publication from the public portal for the purpose of private study or research.

- You may not further distribute the material or use it for any profit-making activity or commercial gain

If the publication is distributed under the terms of Article $25 \mathrm{fa}$ of the Dutch Copyright Act, indicated by the "Taverne" license above, 


\title{
Equilibria with coordination failures
}

\author{
P. Jean-Jacques Herings ${ }^{\mathrm{a}, *}$, Gerard van der Laan ${ }^{\mathrm{b}}$, Dolf Talman ${ }^{\mathrm{c}}$ \\ a Department of Economics, Maastricht University, P.O. Box 616, 6200 MD Maastricht, The Netherlands \\ b Department of Econometrics and Tinbergen Institute, Vrije Universiteit, De Boelelaan 1105, 1081 HV Amsterdam, The Netherlands \\ c Department of Econometrics E' Operations Research and CentER, Tilburg University, P.O. Box 90153, 5000 LE Tilburg, The Netherlands
}

\section{A R T I C L E I N F O}

\section{Article history:}

Received 8 July 2004

Received in revised form 2 February 2007

Accepted 17 November 2007

Available online 6 June 2008

\section{JEL classification:}

C62

C63

C68

D51

\section{Keywords:}

Exchange economy

Rationing

Coordination failures

Price rigidities

\begin{abstract}
A B S T R A C T
This paper extends the literature on equilibria with coordination failures to arbitrary convex sets of admissible prices. This makes it possible to address coordination failures for cases with price indexation or more general price linkages between commodities. We introduce a new equilibrium concept, called quantity constrained equilibrium (QCE), giving a unified treatment to all cases considered in the literature so far. At a QCE the expected trade opportunities on supply and demand are completely determined by a rationing vector satisfying that the prevailing price system maximizes the value of the rationing vector within the set of admissible prices. When the set of admissible prices is compact, we show the existence of a connected set of QCEs. This set connects two trivial no-trade equilibria, one with completely pessimistic expectations concerning supply opportunities and one with completely pessimistic expectations concerning demand opportunities. Moreover, the set contains for every commodity a generalized Drèze equilibrium, being a QCE at which for that commodity no binding trade opportunities on both supply and demand are expected, and also a generalized supply-constrained equilibrium at which no binding constraints on demand opportunities are expected and for at least one commodity also not on supply. We apply this main result to several special cases, and also discuss the case of an unbounded set of admissible prices.
\end{abstract}

(C) 2008 Elsevier B.V. All rights reserved.

\section{Introduction}

This paper is motivated by the renewed interest in equilibria with coordination failures. This interest can be traced back to the work of Roberts (1987a,b, 1989a,b). These papers establish the existence of a continuum of equilibria with quantity rationing of supply at competitive prices in a class of economies characterized by homothetic preferences and constant returns to scale. The important point made in these papers is that rationing is not due to price distortions, but rather to pessimistic expectations concerning trade opportunities. Even at competitive prices, there is a continuum of equilibria with rationing. Rationing is therefore not due to wrong relative prices of commodities but is caused by coordination failures.

Several authors contributed further to the coordination failure literature by generalizing the work of Roberts in a number of directions. Herings (1996a,b, 1998) extends Roberts' results to an exchange economy with standard assumptions on the primitives and allows for non-competitive prices. The set of admissible prices is obtained by specifying, for each commodity, a lower bound and an upper bound on its price. Drèze (1997) and Citanna et al. (2001) treat the combination of fixed and flexible prices and consider the framework of an economy with production. Drèze (2001) focuses on explaining downward real price rigidities and understanding the nature and the sources of the coordination failures. His arguments are based on

\footnotetext{
* Corresponding author.

E-mail addresses: P.Herings@algec.unimaas.nl (P.J.-J. Herings), glaan@econ.vu.nl (G. van der Laan), talman@uvt.nl (D. Talman).
} 
the combination of uncertainty and incomplete markets. When markets are incomplete, price regulations may be used to generate Pareto improvements as stressed also by Drèze and Gollier (1993) and Herings and Polemarchakis (2005).

In this paper we extend the results on equilibria with coordination failures to a setting where the set of admissible prices is not given by a simple combination of fixed and flexible prices or by a simple specification of a lower and an upper bound on the prices of all commodities. We are interested in sets of admissible prices that may reflect any kind of price indexation or price linkages. Special cases of such sets of admissible prices have been studied before in the fix-price literature by Chetty and Nayak (1978), Kurz (1982), Dehez and Drèze (1984), van der Laan (1984) and Weddepohl (1987). To deal with general cases of price restrictions, we allow for an arbitrary convex set of prices and define a unifying equilibrium concept, called quantity constrained equilibrium (QCE). Whereas the literature of the previous paragraph takes an ad hoc approach in defining an equilibrium appropriate for the set of admissible prices studied, we pin down a common principle behind all these definitions. This common principle is that pessimistic expectations concerning supply or demand opportunities may only emerge at a certain price system, if that price system maximizes the value of the rationing vector within the set of admissible prices. An immediate implication of this principle is that pessimistic expectations concerning supply or demand opportunities of a commodity are excluded when the price of that commodity is flexible and that pessimistic expectations concerning supply (demand) possibilities of a commodity may only occur when there is a downwards (upwards) price rigidity.

We show that in case the set of admissible prices is compact and only contains positive price vectors, there exists a continuum of quantity constrained equilibria connecting two trivial equilibria. One trivial QCE is a fully supply-constrained no-trade equilibrium with completely pessimistic expectations concerning supply opportunities for all commodities and prices such that the value of the total initial endowments is minimized within the set of admissible prices. The other trivial QCE is a trivial fully demand-constrained no-trade equilibrium with completely pessimistic expectations concerning demand opportunities for all commodities and prices such that the value of the total initial endowments is maximized.

The connected set of non-trivial QCEs contains for every commodity a generalized Drèze equilibrium, being a QCE at which for that commodity no binding constraints on supply and on demand are expected, a generalized supply-constrained equilibrium, at which no binding constraints on demand are expected and for at least one commodity also not on supply, and a generalized demand-constrained equilibrium, at which no binding constraints on supply are expected and for at least one commodity also not on demand.

In Section 5 we consider some applications and extensions. First we consider the case of price indexation and show that our main theorem extends to the results that were obtained in the literature for special cases of price indexation. Second, we consider cases in which the set of admissible prices is unbounded, allowing for models in which some prices are fully flexible, some prices are bounded, and other prices are linked. In case the set of prices is unbounded, the connected set of QCEs is also unbounded with some of the prices going to infinity, while simultaneously for any commodity with price bounded from above, its relative price tends to zero and eventually all consumers will have complete pessimistic expectations concerning demand opportunities of such a commodity, implying no-trade for this commodity. In the limit the economy reduces to an economy where trade only takes place in the commodities for which the prices are unbounded from above. When the prices of these commodities are not tied to prices of other commodities, these prices tend to Walrasian equilibrium values for this reduced economy. In case this holds for all commodities, there is a connected set of QCEs leading from a fully supply-constrained no-trade equilibrium to a Walrasian equilibrium. Since all consumers keep their initial endowments at a fully supply-constrained equilibrium and the Walrasian equilibrium is Pareto efficient, this case is most striking as far as the potential detrimental effects of coordination failures are concerned.

The paper has been organized as follows. Section 2 describes the model and discusses equilibria with coordination failures in case the prices are fixed or restricted by a lower and an upper bound. Section 3 introduces the general concept of quantity constrained equilibrium. For the compact, convex case with positive prices the existence result is given in Section 4 . In Section 5 we consider the application of general price indexation and the extension to unbounded admissible sets of prices.

\section{Preliminaries}

We consider an exchange economy $\mathcal{E}=\left(\left\{\mathcal{X}^{i}, \succcurlyeq^{i}, w^{i}\right\}_{i=1}^{m}, P\right)$. In this economy there are $m$ consumers, indexed $i=1, \ldots, m$, and $n$ commodities, indexed $j=1, \ldots, n$. For $k$ a positive integer, we denote $I_{k}=\{1, \ldots, k\}$. Each consumer $i \in I_{m}$ is characterized by a consumption set $\mathcal{X}^{i}$, a preference preordering $\succcurlyeq^{i}$ on $\mathcal{X}^{i}$, and a vector of initial endowments $w^{i}$. The total endowment $w$ is defined by $w=\sum_{i \in I_{m}} w^{i}$. We assume that the admissible price systems in the economy $\mathcal{E}$ are described by a set $P \subset \mathbb{R}_{+}^{n}$. We make the following standard assumptions.

Assumption X. For every consumer $i \in I_{m}$, the consumption set $\mathcal{X}^{i}$ is a closed and convex subset of $\mathbb{R}_{+}^{n}$ and $\mathcal{X}^{i}+\mathbb{R}_{+}^{n} \subset \mathcal{X}^{i}$.

Assumption $\mathbf{U}$. For every consumer $i \in I_{m}$, the preference preordering $\succcurlyeq^{i}$ on $\mathcal{X}^{i}$ is complete, continuous, strongly monotonic, and strictly convex. ${ }^{1}$

Assumption W. For every consumer $i \in I_{m}$, the vector of initial endowments $w^{i}$ belongs to the interior of $\mathcal{X}^{i}$.

\footnotetext{
${ }^{1}$ A preference preordering $\succcurlyeq^{i}$ is said to be strongly monotonic if $\bar{x}^{i}, \hat{x}^{i} \in \mathcal{X}^{i}, \bar{x}^{i} \leq \hat{x}^{i}$, and $\bar{x}^{i} \neq \hat{x}^{i}$ implies $\hat{x}^{i} \succ \bar{x}^{i}$. A preference preordering $\succcurlyeq^{i}$ is said to be strictly convex when for any pair $\bar{x}^{i}, \hat{x}^{i} \in \mathcal{X}^{i}$, such that $\bar{x}^{i} \neq \hat{x}^{i}, \bar{x}^{i} \sim \hat{x}^{i}$, it holds that $\lambda \bar{x}^{i}+(1-\lambda) \hat{x}^{i}>\bar{x}^{i}$ for $\lambda \in(0,1)$.
} 
It should be noticed that all results carry over to the case of convex preferences. Assuming strict convexity allows us to work with demand functions instead of demand correspondences and thus simplifies notation. Also the assumption of strongly monotonic preferences is made for the sake of simplicity and can be relaxed considerably.

When trade takes place at some $p \in P$ this does not necessarily imply that $p$ is a competitive equilibrium price system. Therefore there might be markets in excess demand or excess supply. In both cases, a distributional rule is needed to determine the allocation that results. Such a distributional rule is called a rationing system. For example, some households could expect to be rationed on their net demand or net supply. Although we restrict our attention to uniform rationing, i.e. all households that experience supply (demand) rationing in a certain market, will end up with the same net supply (demand) of the commodity concerned, our results carry over to a variety of other rationing systems (see Herings, 1996a for a general treatment of rationing systems). In a uniform rationing system, the expectations of available supply opportunities for a household on the various markets are described by a vector $\ell \in-\mathbb{R}_{+}^{n}$, and the expectations of available demand opportunities by a vector $u \in \mathbb{R}_{+}^{n}$. In equilibrium, the expected trade opportunities are required to be rational and thus match the amounts allocated by the rationing system.

Given a price system $p \in P$ and expected trade opportunities $\ell$ for supply and $u$ for demand, the constrained budget set of consumer $i \in I_{m}$ is given by

$$
B^{i}(p, \ell, u)=\left\{x^{i} \in \mathcal{X}^{i} \mid p \cdot x^{i} \leq p \cdot w^{i} \text { and } \ell_{k} \leq x_{k}^{i}-w_{k}^{i} \leq u_{k}, \forall k \in I_{n}\right\}
$$

and the constrained demand $d^{i}(p, \ell, u)$ of $i$ is the best element for $\succcurlyeq^{i}$ in $B^{i}(p, \ell, u)$. Because of strict convexity and strong monotonicity, this element is unique and lies on the budget hyperplane, i.e. $p \cdot d^{i}(p, \ell, u)=p \cdot w^{i}$.

A well-known particular set $P$ of admissible prices is that $P$ is a cube

$$
C^{n}=\left\{p \in \mathbb{R}_{+}^{n} \mid \underline{p}_{k} \leq p_{k} \leq \bar{p}_{k}, k \in I_{n}\right\},
$$

where $\underline{p}_{k}$ and $\bar{p}_{k}$ are a priorily given lower and upper bounds for the price $p_{k}$ of good $k \in I_{n}$ satisfying $0<\underline{p}_{k} \leq \bar{p}_{k}$. For such a set of admissible prices a constrained equilibrium is defined as follows (Drèze, 1975).

Definition 2.1. A constrained equilibrium for the economy $\mathcal{E}=\left(\left\{\mathcal{X}^{i}, \succcurlyeq^{i}, w^{i}\right\}_{i=1}^{m}, C^{n}\right)$ is a price system $p^{*} \in C^{n}$, expected trade opportunities $\left(\ell^{*}, u^{*}\right) \in-\mathbb{R}_{+}^{n} \times \mathbb{R}_{+}^{n}$, and, for every consumer $i \in I_{m}$, a consumption bundle $x^{* i} \in \mathcal{X}^{i}$ such that

(i) for all $i \in I_{m}, x^{* i}=d^{i}\left(p^{*}, \ell^{*}, u^{*}\right)$;

(ii) $\sum_{i=1}^{m} x^{* i}=w$;

(iii) for all $k \in I_{n}: x_{k}^{* h}-w_{k}^{h}=\ell_{k}^{*}$ for some $h \in I_{m}$ implies $x_{k}^{* i}-w_{k}^{i}<u_{k}^{*}, \forall i \in I_{m}$, and, analogously, $x_{k}^{* h}-w_{k}^{h}=u_{k}^{*}$ for some $h \in I_{m}$ implies $x_{k}^{* i}-w_{k}^{i}>\ell_{k}^{*}, \forall i \in I_{m}$

(iv) for all $k \in I_{n}$, if there is $i \in I_{m}$ such that $\ell_{k}^{*}=x_{k}^{* i}-w_{k}^{i}$, then $p_{k}^{*}=\underline{p}_{k}$ and if there is $i \in I_{m}$ such that $u_{k}^{*}=x_{k}^{* i}-w_{k}^{i}$, then $p_{k}^{*}=\bar{p}_{k}$.

Condition (i) requires that the consumption of each consumer equals his constrained demand, while Condition (ii) is the market clearing condition. Condition (iii) says that markets are frictionless, i.e. there can not be (binding) pessimistic expectations simultaneously on both sides of a market. Condition (iv) precludes pessimistic expectations concerning supply (demand respectively) opportunities in the market of some commodity as long as its price is not on its lower (upper) bound. Herings (1998) has shown that there exists a connected set of (non-trivial) constrained equilibria that connects two trivial equilibria. Both trivial equilbria are extreme cases of coordination failure. One is the fully supply-constrained equilibrium given by $\ell^{*}=0^{n}, p^{*}=p$ and $x^{* i}=w^{i}, i \in I_{m}$, i.e. each consumer has completely pessimistic expectations about his supply opportunities. The other is the fully demand-constrained equilibrium with completely pessimistic expectations about demands, given by $u^{*}=0^{n}, p^{*}=\bar{p}$ and $x^{* i}=w^{i}, i \in I_{m}$. Because of the completely pessimistic expectations, in the fully supplyconstrained (demand-constrained, respectively) equilibrium no consumer expresses any demand (supply) and therefore the completely pessimistic expectations are confirmed. The continuum result of Herings (1998) admits very simple proofs of a number of special cases, like the existence of a Drèze equilibrium with respect to any a priorily chosen commodity (Drèze, 1975) and the existence of a supply-constrained equilibrium (van der Laan, 1980, 1982). A Drèze equilibrium with respect to commodity $k$ is a constrained equilibrium without rationing in the market for commodity $k$. A supply-constrained equilibrium is a constrained equilibrium without rationing on any demand and without rationing on the market of at least one commodity. An algorithm to compute the connected set of constrained equilibria has been proposed in Herings et al. (1996). For results on the set of equilibria when $p_{k}=\bar{p}_{k}$ for all $k \in I_{n}$ and thus the set $P=C^{n}$ reduces to a single element, we refer to for instance Herings (1998) or Citanna et al. (2001).

\section{Quantity constrained equilibria}

An equilibrium concept for a general set $P$ of price restrictions should contain the concept of Definition 3.1 as a special case when $P=C^{n}$. The utility maximizing Condition (i) and the market clearing Condition (ii) of Definition 2.1 carry over easily to the general case. Also Condition (iii) that markets are frictionless is not difficult to implement. Difficulties only arise in generalizing Condition (iv). This condition says that pessimistic expectations are only allowed when the price mechanism of falling prices in case of excess supply and rising prices in case of excess demand is not able to operate because of the lower 
and upper bounds. To generalize this condition to general sets $P$ we will allow that pessimistic expected trade opportunities take over and may constrain individual excess demand or supply accordingly, when prices can not be adjusted in such a way that the unconstrained excess demands and supplies are expected to decrease in absolute value. To make this more precise, we first reformulate Definition 2.1.

It should be noticed that different expected supply and demand opportunities may yield the same constrained equilibrium allocation. When expectations concerning supply (demand respectively) opportunities are not binding, the precise specification of the expected supply opportunity $\ell_{k}^{*}$ (demand opportunity $u_{k}^{*}$ ) is immaterial, as long as $\ell_{k}^{*}<x_{k}^{* i}-w_{k}^{i}\left(u_{k}^{*}>x_{k}^{* i}-w_{k}^{i}\right.$ ) for all $i \in I_{m}$. This freedom in the specification of non-binding opportunities can be used to simplify the equilibrium definition by making a particular choice. In the sequel we focus on expected trade opportunities $(l, u)$ that are represented by a single vector $r \in[-w, w]$, called the rationing vector, where $[-w, w]=\left\{x \in \mathbb{R}^{n} \mid-w \leq x \leq w\right\}$. A rationing vector $r \in[-w, w]$ induces expected trade opportunities $(\ell, u)$ given by

$$
\ell=-w-r \leq 0^{n} \quad \text { and } \quad u=w-r \geq 0^{n} .
$$

Making use of $r$ gives the following redefinition of constrained equilibrium on $C^{n}$

Definition 3.1. A constrained equilibrium for the economy $\mathcal{E}=\left(\left\{\mathcal{X}^{i}, \succcurlyeq^{i}, w^{i}\right\}_{i=1}^{m}, C^{n}\right)$ is a price system $p^{*} \in C^{n}$, a rationing vector $r^{*} \in[-w, w]$, and, for every consumer $i \in I_{m}$, a consumption bundle $\mathcal{X}^{* i} \in \mathcal{X}^{i}$ such that

(i) for all $i \in I_{m}, x^{* i}=d^{i}\left(p^{*},-w-r^{*}, w-r^{*}\right)$;

(ii) $\sum_{i=1}^{m} x^{* i}=w$;

(iii) for all $k \in I_{n}$, if there is $i \in I_{m}$ such that $-w_{k}-r_{k}^{*}=x_{k}^{* i}-w_{k}^{i}$, then $p_{k}^{*}=\underline{p}_{k}$ and if there is $i \in I_{m}$ such that $w_{k}-r_{k}^{*}=x_{k}^{* i}-w_{k}^{i}$, then $p_{k}^{*}=\bar{p}_{k}$.

Since $0^{n} \leq x^{* i} \leq w$ and $0^{n} \ll w^{i} \ll w$ and therefore $-w \ll x^{* i}-w^{i} \ll w$ for any $i$, a negative value of $r_{k}^{*}$ implies that only expected trade opportunities concerning the supply of commodity $k$ can be binding, in which case $p_{k}$ is on its lower bound, while the opposite holds in case of a positive value of $r_{k}^{*}$. When $r_{k}^{*}=0$, then expected trade opportunities on neither the demand side nor the supply side of the market for commodity $k$ can be binding. Therefore Condition (iii) replaces both Condition (iii) and Condition (iv) of Definition 2.1.

A constrained equilibrium according to Definition 3.1 has the property that the equilibrium rationing vector $r^{*}$ satisfies $p^{*} \cdot r^{*}=\max \left\{p \cdot r^{*} \mid p \in C^{n}\right\}$, i.e. $r^{*}$ points outwards to $C^{n}$ at the equilibrium price vector $p^{*}$. We use this property to generalize Definition 3.1 to general price sets $P$. Formally, we require that at a constrained equilibrium the rationing vector $r \in[-w, w]$ is such that $p \cdot r=\max \{\hat{p} \cdot r \mid \hat{p} \in P\}$ and thus points outwards to $P$ at $p$. In the sequel a pair $(p, r)$ satisfying this property will be called stable. A stable equilibrium pair $\left(p^{*}, r^{*}\right)$ implies that rationing is not binding when $p^{*}$ lies in the interior of the set $P$, because then stability implies that $r^{*}=0^{n}$. Indeed, when $p^{*}$ lies in the interior of $P$, prices are fully flexible and can be adjusted according to the law of supply and demand and therefore excess demand or excess supply need not be constrained by rationing. If $p^{*}$ does not lie in the interior of the set $P$, then the stability property implies that the rationing vector $r^{*}$ points outwards to $P$ at $p^{*}$, being a direction into which the price vector cannot be further adjusted. The collection of all these directions is called the normal cone at $p^{*}$ to $P$. More formally, for $p \in P$, the normal cone $G(p)$ to $P$ at $p$ is given by

$$
G(p)=\left\{y \in \mathbb{R}^{n} \mid \hat{p} \cdot y \leq p \cdot y \text { for any } \hat{p} \in P\right\}
$$

and determines precisely the rationing vectors that may occur at $p$. The stability concept is therefore equivalent to the requirement that $r \in G(p)$. This gives us the following definition of the general concept of a quantity constrained equilibrium.

Definition 3.2. (quantity constrained equilibrium) A quantity constrained equilibrium for the economy $\mathcal{E}=$ $\left(\left\{\mathcal{X}^{i}, \succcurlyeq^{i}, w^{i}\right\}_{i=1}^{m}, P\right)$ is a price system $p^{*} \in P$, a rationing vector $r^{*} \in[-w, w]$, and, for every consumer $i \in I_{m}$, a consumption bundle $x^{* i} \in \mathcal{X}^{i}$ satisfying

(i) for all $i \in I_{m}, x^{* i}=d^{i}\left(p^{*},-w-r^{*}, w-r^{*}\right)$;

(ii) $\sum_{i=1}^{m} x^{* i}=w$;

(iii) $r^{*} \in G\left(p^{*}\right)$.

Condition (iii) links the rationing vector to the price restrictions. Expected trade opportunities are completely determined by a vector $r^{*}$ in the normal cone $G\left(p^{*}\right)$ to the set of admissible prices $P$ at the equilibrium price system $p^{*}$. In case that $P$ equals $C^{n}$, consider a pair $\left(p^{*}, r^{*}\right)$ with $r_{k}^{*}$ positive. Then such a pair can only be stable when $p_{k}^{*}=\bar{p}_{k}$. Similarly, when $r_{k}^{*}$ is negative, the pair can only be stable when $p_{k}^{*}=\underline{p}_{k}$. Therefore, in this case the requirement that ( $\left.p^{*}, r^{*}\right)$ be stable yields precisely Condition (iii) of Definition 3.1.

In general, since $0^{n} \leq x^{* i} \leq w$ and $0^{n} \ll w^{i} \ll w$, in a QCE it holds that $-w_{k}<x_{k}^{* i}-w_{k}^{i}<w_{k}$ for all $k \in I_{n}$ and $i \in I_{m}$. Hence, in a QCE, a consumer $i \in I_{m}$ can only face binding expected demand opportunities for good $k$ if $r_{k}^{*}=w_{k}-\left(x_{k}^{* i}-w_{k}^{i}\right)>0$. Analogously, some consumer $i \in I_{m}$ can only face binding expected supply opportunities for good $k$ if $r_{k}^{*}=-w_{k}-\left(x_{k}^{* i}-w_{k}^{i}\right)<$ 0 . Notice that the more the expectations concerning supply (demand) opportunities of commodity $k$ are pessimistic, the 
closer its rationing level $r_{k}^{*}$ is to $-w_{k}$ (to $w_{k}$ ). In particular, expectations concerning supply opportunities of commodity $k$ are completely pessimistic if $r_{k}^{*}=-w_{k}$ and expectations concerning demand opportunities of commodity $k$ are completely pessimistic if $r_{k}^{*}=w_{k}$. When $p^{*}$ is in the interior of $P$, then $r^{*}=0^{n}$, in which case the expected demand opportunities equal $w$ and the expected supply opportunities equal $-w$, implying that in equilibrium the expected trade opportunities are not binding and thus $p^{*}$ is a Walrasian equilibrium price vector. Summarizing, in a QCE consumers maximize their utility in their constrained budget sets, total demand equals total supply, there are no binding expected trade opportunities on both supply and demand simultaneously, and the rationing vector points into a direction such that the value of the rationing vector can not be increased by moving the price vector within $P$.

From an economic point of view, it is of crucial importance that the equilibrium concept is independent of the units of measurement that are used in the definition of a commodity. Suppose that the unit of measurement used in the definition of commodity $k$ is multiplied by a positive constant $\alpha_{k}, k=1, \ldots, n$. Let $\alpha=\left(\alpha_{1}, \ldots, \alpha_{n}\right)$. An economy $\mathcal{E}(\alpha)$ with initial endowments $w^{i}(\alpha)$ given by $w_{k}^{i}(\alpha)=w_{k} / \alpha_{k}, k=1, \ldots, n$, set of admissible prices

$$
P(\alpha)=\left\{\hat{p} \in \mathbb{R}^{n} \mid \hat{p}_{k}=\alpha_{k} p_{k}, \quad k=1, \ldots, n, \text { for somep } \in P\right\}
$$

and appropriately redefined consumption sets $\mathcal{X}^{i}(\alpha)$ and preference relations $\succcurlyeq^{i}(\alpha)$ should have an equivalent set of QCEs as the economy $\mathcal{E}$. More precisely, for each QCE $\left(p^{*}, r^{*}, x^{*}\right)$ of $\mathcal{E}$ there should be a $\mathrm{QCE}\left(p^{*}(\alpha), r^{*}(\alpha), x^{*}(\alpha)\right)$ of $\mathcal{E}(\alpha)$ and vice versa, where $p^{*}(\alpha)$ is obtained from $p^{*}$ by componentwise multiplication by $\alpha$, and $r^{*}(\alpha)$ and $x^{*}(\alpha)$ are obtained from $r^{*}$ and $x^{*}$ by componentwise division by $\alpha$. The following result claims that equivalence indeed holds.

Theorem 3.3. For any choice of $\alpha \gg 0$, it holds that $\left(p^{*}, r^{*}, x^{*}\right)$ is a QCE of $\mathcal{E}=\left(\left\{\mathcal{X}^{i}, \succcurlyeq^{i}, w^{i}\right\}_{i=1}^{m}, P\right)$ if and only if $\left(p^{*}(\alpha), r^{*}(\alpha), x^{*}(\alpha)\right)$ is a $Q C E$ of $\mathcal{E}(\alpha)=\left(\left\{\mathcal{X}^{i}(\alpha), \succcurlyeq^{i}(\alpha), w^{i}(\alpha)\right\}_{i=1}^{m}, P(\alpha)\right)$.

Proof. It is obvious that $\left(p^{*}, r^{*}, x^{*}\right)$ satisfies Conditions (i) and (ii) of Definition 3.2 of a QCE of $\mathcal{E}$ if and only if $\left(p^{*}(\alpha), r^{*}(\alpha), x^{*}(\alpha)\right)$ satisfies Conditions (i) and (ii) of Definition 3.2 of a QCE of $\mathcal{E}(\alpha)$. It is easily verified that $r^{*} \in G\left(p^{*}\right)$ if and only if $r^{*}(\alpha) \in G^{\alpha}\left(p^{*}(\alpha)\right)$, where $G^{\alpha}\left(p^{*}(\alpha)\right)$ denotes the normal cone to $P(\alpha)$ at $p^{*}(\alpha)$. This shows that $\left(p^{*}, r^{*}, x^{*}\right)$ satisfies Condition (iii) of the definition of a QCE of $\mathcal{E}$ if and only if $\left(p^{*}(\alpha), r^{*}(\alpha), x^{*}(\alpha)\right)$ satisfies Condition (iii) of the definition of a QCE of $\mathcal{E}(\alpha)$.

\section{Existence results}

In this section we consider the case that $P$ is a compact, convex set containing only positive prices. In the next section we also allow for an unbounded set of admissible prices.

Assumption P. The set $P$ of admissible prices is a non-empty, convex, and compact subset in the interior of $\mathbb{R}_{+}^{n}$.

We first show that there are two different trivial no-trade QCEs, one with completely pessimistic expectations concerning supply opportunities, implied by $r^{*}=-w$, and one with completely pessimistic expectations concerning demand opportunities, implied by $r^{*}=w$. Let $P^{0}$ and $P^{1}$ be given by

$$
P^{0}=\{p \in P \mid w \cdot p \leq w \cdot \tilde{p} \text { for all } \tilde{p} \in P\}
$$

and

$$
P^{1}=\{p \in P \mid w \cdot p \geq w \cdot \tilde{p} \text { for all } \tilde{p} \in P\} .
$$

Under Assumption $P$, both $P^{0}$ and $P^{1}$ are non-empty, convex and compact and their intersection is either empty or equal to $P$. Prices in $P^{0}$ are such that the value of the total initial endowment $p \cdot w$ is minimized, and prices in $P^{1}$ are such that this value is maximized. The next result claims that there is a trivial no-trade QCE with completely pessimistic expectations concerning supply opportunities at any price in $P^{0}$ and a trivial no-trade QCE with completely pessimistic expectations concerning demand opportunities at any price in $P^{1}$.

Theorem 4.1. For any $p \in P^{0}$ there is a fully supply-constrained equilibrium with rationing vector $r^{*}=-w$ and allocation $x^{* i}=$ $w^{i}, i \in I_{m}$. For any $p \in P^{1}$ there is a fully demand-constrained equilibrium with rationing vector $r^{*}=w$ and allocation $x^{* i}=w^{i}, i \in I_{m}$.

Proof. Take any $p \in P^{0}$. By definition of $P^{0}$ we have that $-w \in G(p)$. Taking $r=-w$ gives for any $i \in I_{m}$ that $B^{i}\left(p, 0^{n}, 2 w\right)=\left\{w^{i}\right\}$ and hence $d^{i}\left(p, 0^{n}, 2 w\right)=w^{i}$, implying that markets clear and thus a no-trade equilibrium with completely pessimistic expectations concerning supply opportunities is obtained.

Analogously, take any $p \in P^{1}$. It holds that $w \in G(p)$. Taking $r=w$, we have for any $i \in I_{m}$ that $B^{i}\left(p,-2 w, 0^{n}\right)=\left\{x^{i} \in \mathcal{X}^{i} \mid x^{i} \leq w^{i}\right\}$ and hence by Assumption $U$ that $d^{i}\left(p,-2 w, 0^{n}\right)=w^{i}$, implying that markets clear and thus a no-trade equilibrium with completely pessimistic expectations concerning demand opportunities is obtained.

If at a QCE it holds that $r^{*}=-w$ or $r^{*}=w$, we say that the QCE is trivial. Otherwise, we call the QCE non-trivial. The existence of a continuum of non-trivial QCEs follows from the next result, which says that there exists a connected set of QCEs containing both a trivial no-trade equilibrium with completely pessimistic expectations concerning supply opportunities and a trivial no-trade equilibrium with completely pessimistic expectations concerning demand opportunities. 
Theorem 4.2. Let $\mathcal{E}=\left(\left\{\mathcal{X}^{i}, \succcurlyeq^{i}, w^{i}\right\}_{i=1}^{m}, P\right)$ be an economy satisfying Assumptions $\mathrm{X}, \mathrm{U}, \mathrm{W}$ and $\mathrm{P}$. Then there exists a connected set $D$ of quantity constrained equilibria of the economy $\mathcal{E}$, containing a fully supply-constrained equilibrium $\left(p^{0},-w, w^{1}, \ldots, w^{m}\right)$ for some $p^{0} \in P^{0}$, and a fully demand-constrained equilibrium $\left(p^{1}, w, w^{1}, \ldots, w^{m}\right)$ for some $p^{1} \in P^{1}$.

Since the rationing vector is equal to $-w$ at a fully supply-constrained equilibrium and equal to $w$ at a fully demandconstrained equilibrium, the connected set $D$ contains a continuum of non-trivial QCEs.

We continue this section with the proof of Theorem 4.2 and conclude the section with a discussion of the implications of the result. We first focus on the equilibrating mechanism to find a QCE. To do so, we introduce a set $Q \subset \mathbb{R}^{n}$ containing $P$ and define for every $q \in Q$ an admissible price vector $p(q) \in P$ and a rationing vector $r(q) \in[-w, w]$. The set $Q$ is taken to be

$$
Q=\left\{q \in \mathbb{R}^{n} \mid\|q-p\|_{2} \leq 1 \text { for some } p \in P\right\},
$$

so $Q$ is the set of elements in $\mathbb{R}^{n}$ lying at most at a distance 1 from $P$, using the Euclidean norm, and thus includes the set $P$. In the sequel bnd $(Q)$ denotes the boundary of $Q$ and $\operatorname{int}(Q)$ its interior. For any $q \in Q$, we define the corresponding price vector $p(q)$ to be the projection of $q$ on $P$, i.e.

$$
p(q)=\arg \min _{p \in P}\|p-q\|_{2} .
$$

Since by Assumption P, the set $P$ is convex and compact, for every $q \in Q$ it holds that $p(q)$ is uniquely defined and is continuous in $q$, and $q-p(q) \in G(p(q))$. Moreover, $\|q-p(q)\|_{2} \leq 1$, with equality if and only if $q \in$ bnd $(Q)$. It also holds that $p(q)=q$ if and only if $q \in P$. The set $Q$ has the following properties.

\section{Lemma 4.3.}

(i) The set $Q$ is a convex, compact, full-dimensional subset of $\mathbb{R}^{n}$ and contains $P$ in its interior;

(ii) The boundary of $Q$ is smooth.

Proof. The compactness follows from the compactness of $P$. That $Q$ is full-dimensional and contains $P$ in its interior follows immediately from its definition. To prove convexity, take any $q^{1}, q^{2} \in Q$ and $0 \leq \lambda \leq 1$, and let $q(\lambda)=\lambda q^{1}+(1-\lambda) q^{2}$ and $p(\lambda)=\lambda p\left(q^{1}\right)+(1-\lambda) p\left(q^{2}\right)$. Since $P$ is convex, we know that $p(\lambda) \in P$. Moreover, $\|q(\lambda)-p(\lambda)\|_{2} \leq \lambda\left\|q^{1}-p\left(q^{1}\right)\right\|_{2}+(1-$ $\lambda)\left\|q^{2}-p\left(q^{2}\right)\right\|_{2} \leq 1$. Therefore, $q(\lambda) \in Q$.

Property (ii) follows from the use of the 2 -norm in the definition of $Q$. Take any $q^{*} \in \operatorname{bnd}(Q)$. Then $\left\|q^{*}-p\left(q^{*}\right)\right\|_{2}=1$ and any $q$ with $\left\|q-p\left(q^{*}\right)\right\|_{2} \leq 1$ belongs to $Q$. Hence, the normal cone at $q^{*}$ contains at most one vector with length one. Since $Q$ is convex, we know that the normal cone at $q^{*}$ is non-empty and upper-semi-continuous. Hence, the normal cone at any boundary point $q$ of $Q$ contains a unique vector with length one and this vector is continuous in $q$, i.e. the boundary of $Q$ is smooth.

We define the rationing vector $r(q)$ corresponding to $q$ by the function $r: Q \rightarrow \mathbb{R}^{n}$, where

$$
r(q)=0^{n}, \quad q \in P,
$$

and

$$
r(q)=\min _{j \in I_{n}} \frac{w_{j}}{\left|q_{j}-p_{j}(q)\right|}\|q-p(q)\|_{2}(q-p(q)), \quad q \in Q \backslash P .
$$

Since $\|q-p(q)\|_{2}$ goes to zero when $q-p(q)$ converges to $0^{n}$ and, for all $k \in I_{n}$,

$$
-w_{k} \leq\left(\min _{j \in I_{n}} \frac{w_{j}}{\left|q_{j}-p_{j}(q)\right|}\right)\left(q_{k}-p_{k}(q)\right) \leq w_{k},
$$

it follows that $r(q)$ goes to $0^{n}$ when $q$ converges to a point in $P$. This implies that $r(q)$ is continuous in $q$. The other properties below immediately follow from the definition of the function $r$.

Corollary 4.4. The function $r: Q \rightarrow \mathbb{R}^{n}$ satisfies the following properties:

(i) $r$ is continuous;

(ii) $r(q) \in[-w, w]$ for all $q \in Q$;

(iii) $r_{k}(q)=w_{k}$ if and only if $w_{k} /\left(q_{k}-p_{k}(q)\right)=\min _{j \in I_{n}} w_{j} /\left(\left|q_{j}-p_{j}(q)\right|\right)$ and $q \in$ bnd(Q);

(iv) $r_{k}(q)=-w_{k}$ if and only if $-w_{k} /\left(q_{k}-p_{k}(q)\right)=\min _{j \in I_{n}} w_{j} /\left(\left|q_{j}-p_{j}(q)\right|\right)$ and $q \in$ bnd(Q);

(v) $r(q) \in G(p(q))$ for all $q \in Q$.

We now define for any consumer $i \in I_{m}$ the reduced budget correspondence $B^{i}: Q \rightarrow \mathbb{R}^{n}$ by

$$
B^{i}(q)=B^{i}(p(q),-w-r(q), w-r(q)), \quad q \in Q .
$$


Finally, for any consumer $i \in I_{m}$ we define the reduced demand function $d^{i}: Q \rightarrow \mathbb{R}^{n}$ by $d^{i}(q)=d^{i}(p(q),-w-r(q), w-r(q))$. Because the set $P$ is in the interior of $\mathbb{R}_{+}^{n}$, we have that for any $q \in Q$ the price vector $p(q) \in P$ is strictly positive. Notice that since $-w-r(q)$ can be equal to zero, the cheaper point assumption that is usually required to show continuity of the budget correspondence, is violated. Nevertheless, it follows from Theorem 2.2 in Herings (1996b) that $B^{i}$ is continuous at any $q \in Q$. With Assumption $U$ it then follows that $d^{i}$ is a continuous function and so is the reduced excess demand function $z: Q \rightarrow \mathbb{R}^{n}$ defined by

$$
z(q)=\sum_{i \in I_{m}} d^{i}(q)-w
$$

By Assumption $U$ the budget constraint $p(q) \cdot d^{i}(q) \leq p(q) \cdot w^{i}$ is always satisfied with equality and hence Walras' law holds, i.e. $p(q) \cdot z(q)=0$ for all $q \in Q$. The function $z$ satisfies the following properties.

Lemma 4.5. Under Assumptions $\mathrm{X}, \mathrm{U}, \mathrm{W}$ and $\mathrm{P}$, the reduced excess demand function $z: Q \rightarrow \mathbb{R}^{n}$ satisfies the following:

(i) $z$ is continuous;

(ii) Walras' law holds: $p(q) \cdot z(q)=0$ for all $q \in Q$;

(iii) $r_{k}(q)=-w_{k}$ implies $z_{k}(q) \geq 0$ and $r_{k}(q)=w_{k}$ implies $z_{k}(q) \leq 0$.

The next lemma shows that a zero point of $z$ on $Q$ induces a QCE.

Lemma 4.6. Let $q^{*}$ be a zero point of $z$ on $Q$ i.e. $z\left(q^{*}\right)=0^{n}$. Then $\left(p\left(q^{*}\right), r\left(q^{*}\right), d^{1}\left(q^{*}\right), \ldots, d^{m}\left(q^{*}\right)\right)$ is a quantity constrained equilibrium.

Proof. We have to show that Conditions (i)-(iii) of Definition 3.2 hold. Clearly, $p\left(q^{*}\right) \in P, r\left(q^{*}\right) \in[-w, w]$, and Conditions (i) and (ii) hold by construction of the reduced excess demand function. By Property (v) of Corollary 4.4 we have that $r\left(q^{*}\right)$ is an element of $G\left(p\left(q^{*}\right)\right)$, which shows that Condition (iii) of Definition 3.2 holds.

From Lemma 4.6 it follows that the questin of existence of a QCE reduces to the existence of a zero point of $z$. Before stating our general result on the connected set of equilibria in terms of the parameter $q$, we first show that there are two different kinds of trivial zero points of $z$, one corresponding to a trivial no-trade QCE with completely pessimistic expectations concerning supply opportunities and one with completely pessimistic expectations concerning demand opportunities. Let $Q^{0}$ and $Q^{1}$ be given by

$$
Q^{0}=\{q \in Q \mid w \cdot q \leq w \cdot \tilde{q} \text { for all } \tilde{q} \in Q\}
$$

and

$$
Q^{1}=\{q \in Q \mid w \cdot q \geq w \cdot \tilde{q} \text { for all } \tilde{q} \in Q\}
$$

Clearly, since $Q$ is compact, the sets $Q^{0}$ and $Q^{1}$ are both non-empty. Moreover, the intersection of $Q^{0}$ and $Q^{1}$ is empty, since $Q$ is full-dimensional.

Lemma 4.7. Each element in $Q^{0}$ or $Q^{1}$ is a zero point of $z$ and yields a trivial no-trade quantity constrained equilibrium:

(i) Any $q \in Q^{0}$ induces the fully supply-constrained $Q C E\left(p(q),-w, w^{1}, \ldots, w^{m}\right)$ with $p(q) \in P^{0}$.

(ii) Any $q \in Q^{1}$ induces the fully demand-constrained $Q C E\left(p(q), w, w^{1}, \ldots, w^{m}\right)$ with $p(q) \in P^{1}$.

Proof. Take any $q \in Q^{0}$. Clearly, $q \in$ bnd $(Q)$. By definition of $Q^{0}$ we have that $r(q)=-w$ and $p(q) \in P^{0}$. It follows that, for any $i \in I_{m}, B^{i}(q)=\left\{w^{i}\right\}$ and hence $d^{i}(q)=w^{i}$, implying that $z(q)=0^{n}$ and thus $q$ induces a no-trade equilibrium with completely pessimistic expected supply opportunities.

Analogously, take any $q \in Q^{1}$. Then $r(q)=w$ and $p(q) \in P^{1}$. It follows that, for any $i \in I_{m}, B^{i}(q)=\left\{x^{i} \in \mathcal{X}^{i} \mid x^{i} \leq w^{i}\right\}$ and hence by Assumption $U$ that $d^{i}(q)=w^{i}$, implying that $z(q)=0^{n}$ and thus $q$ induces a no-trade equilibrium with completely pessimistic expected demand opportunities.

From this lemma it follows immediately that all trivial QCEs are induced by $Q^{0}$ and $Q^{1}$ and that every zero point of $z$ not in $Q^{0}$ or $Q^{1}$ induces a non-trivial QCE. Theorem 4.2 now follows from the next result saying that $Q$ contains a connected set $C$ of zero points of $z$ having a non-empty intersection with both $Q^{0}$ and $Q^{1}$.

Theorem 4.8. Let $\mathcal{E}=\left(\left\{\mathcal{X}^{i}, \succcurlyeq^{i}, w^{i}\right\}_{i=1}^{m}, P\right)$ be an economy satisfying Assumptions $\mathrm{X}, \mathrm{U}, \mathrm{W}$ and $\mathrm{P}$. Then there exists a connected set $C \subset Q$ of zero points of $z$ such that $C \cap Q^{0} \neq \emptyset$ and $C \cap Q^{1} \neq \emptyset$. 
Proof. Let $\gamma_{0}$ and $\gamma_{1}$ be such that $\sum_{k=1}^{n} w_{k} q_{k}=\gamma_{0}$ when $q \in Q^{0}$ and $\sum_{k=1}^{n} w_{k} q_{k}=\gamma_{1}$ when $q \in Q^{1}$. Notice that $\gamma_{0}$ and $\gamma_{1}$ are well-defined, $\gamma_{1}>\gamma_{0}$ and $\gamma_{0} \leq \sum_{k=1}^{n} w_{k} q_{k} \leq \gamma_{1}$ for all $q \in Q$. For some $M>0$, define $X^{0} \subset \mathbb{R}^{n}$ by

$$
X^{0}=\left\{x \in \mathbb{R}^{n} \mid \sum_{k=1}^{n} w_{k} x_{k}=\gamma_{0}, \max _{k \in I_{n}} x_{k} \leq M\right\}
$$

and, for $0<\alpha \leq 1, X^{\alpha} \subset \mathbb{R}^{n}$ by

$$
X^{\alpha}=\left\{x \in \mathbb{R}^{n} \mid x=x^{0}+\frac{\alpha}{\sum_{k=1}^{n} w_{k}^{2}}\left(\gamma_{1}-\gamma_{0}\right) w, x^{0} \in X^{0}\right\} .
$$

Clearly, for $x \in X^{\alpha}, 0 \leq \alpha \leq 1$, we have that $\sum_{k=1}^{n} w_{k} x_{k}=(1-\alpha) \gamma_{0}+\alpha \gamma_{1}$, and thus $\sum_{k=1}^{n} w_{k} x_{k}=\gamma_{0}$ when $x \in X^{0}$ and $\sum_{k=1}^{n} w_{k} x_{k}=\gamma_{1}$ when $x \in X^{1}$. Further, define

$$
X=\cup_{\alpha \in[0,1]} X^{\alpha}
$$

and take $M$ sufficiently large that $Q \subset X$ and that any $q \in Q \backslash\left(Q^{0} \cup Q^{1}\right)$ lies in the interior of $X$. Notice that $Q^{0}=Q \cap X^{0}$ and $Q^{1}=Q \cap X^{1}$.

We define the set $\bar{X}^{0}=\left\{x \in \mathbb{R}^{n} \mid \sum_{k=1}^{n} w_{k} x_{k}=\gamma_{0}\right\}$. Let $\tau(x)$ be the orthogonal projection of $x \in \mathbb{R}^{n}$ on $\bar{X}^{0}$, i.e. $\tau(x)=x-\lambda w$ for some $\lambda \in \mathbb{R}$. Next, let the set $\tilde{X}^{0}$ be defined by

$$
\tilde{X}^{0}=\left\{x \in \bar{X}^{0} \mid x=\tau(q+z(q)), q \in Q\right\} \cup\left\{x \in \bar{X}^{0} \mid x=\tau(p(q)), q \in \operatorname{bnd}(Q)\right\} .
$$

Since $Q$ is compact and $z$ and $\tau$ are continuous functions, it follows that $\tilde{X}^{0}$ is a bounded subset of $\bar{X}^{0}$ and thus $M$ can be taken so large that $X^{0}$ contains $\tilde{X}^{0}$ in its relative interior. by

For $x \in X^{0}$ and $\alpha \in[0,1]$, denote $x^{\alpha}=x+\frac{\alpha}{\sum_{k=1}^{n} w_{k}^{2}}\left(\gamma_{1}-\gamma_{0}\right) w \in X^{\alpha}$ and define the point-to-set $\operatorname{mapping} \varphi: X^{0} \times[0,1] \rightarrow X^{0}$

$$
\varphi(x, \alpha)=\left\{\begin{array}{l}
\left\{\tau\left(x^{\alpha}+z\left(x^{\alpha}\right)\right)\right\} \text { if } x^{\alpha} \in \operatorname{int}(Q), \\
\operatorname{Conv}\left(\left\{\tau\left(x^{\alpha}+z\left(x^{\alpha}\right)\right)\right\} \cup\left\{\tau\left(p\left(x^{\alpha}\right)\right)\right\}\right) \text { if } x^{\alpha} \in \operatorname{bnd}(Q), \\
\left\{\tau\left(p\left(q\left(x^{\alpha}\right)\right)\right)\right\} \text { if } x^{\alpha} \in X \backslash Q,
\end{array}\right.
$$

for all $(x, \alpha) \in X^{0} \times[0,1]$, where Conv $(\cdot)$ denotes the convex hull of a set and $q(x)$ is the orthogonal projection of $x \in \mathbb{R}^{n}$ on $Q$. Clearly, $X^{0}$ is compact and convex, the mapping $\varphi$ is upper semi-continuous, and for every $(x, \alpha) \in X^{0} \times[0,1]$ it holds that $\varphi(x, \alpha)$ is compact, convex and non-empty. According to Browder's theorem, see Browder (1960), there exists a connected set $\bar{C}$ of fixed points of $\varphi$ in $X^{0} \times[0,1]$ such that $\bar{C} \cap\left(X^{0} \times\{0\}\right) \neq \emptyset$ and $\bar{C} \cap\left(X^{0} \times\{1\}\right) \neq \emptyset$, i.e. there exists a connected set $\bar{C}$ in $X^{0} \times[0,1]$ satisfying

$$
x \in \varphi(x, \alpha) \text {,for all }(x, \alpha) \in \bar{C},
$$

containing both a point in $X^{0} \times\{0\}$ and a point in $X^{0} \times\{1\}$. Hence, the set $C \subset X$ defined by

$$
C=\left\{\bar{x} \in X \mid \bar{x}=x^{\alpha},(x, \alpha) \in \bar{C}\right\}
$$

is a connected set in $X$ such that $C \cap X^{0} \neq \emptyset$ and $C \cap X^{1} \neq \emptyset$. It remains to be shown that every element of $C$ lies in $Q$ and is a zero point of $z$.

Take any $\bar{x} \in C$, then $\bar{x}=x^{\alpha}$ for some $(x, \alpha) \in \bar{C}$. Suppose first that $x^{\alpha} \in X \backslash Q$. From the definition of $\varphi$ it then follows that

$$
x=\tau\left(p\left(q\left(x^{\alpha}\right)\right)\right) \text {. }
$$

Since $\tau(\bar{x})$ is the projection of $\bar{x}$ on $X^{0}$, there exists $\lambda \in \mathbb{R}$ such that $x=\tau\left(p\left(q\left(x^{\alpha}\right)\right)\right)=p\left(q\left(x^{\alpha}\right)\right)-\lambda w$. Since $x^{\alpha}-q\left(x^{\alpha}\right)=$ $\beta\left(q\left(x^{\alpha}\right)-p\left(q\left(x^{\alpha}\right)\right)\right)$ for some $\beta \geq 0$, it follows that

$$
\begin{aligned}
(\beta+1)\left(q\left(x^{\alpha}\right)-p\left(q\left(x^{\alpha}\right)\right)\right) & =x^{\alpha}-p\left(q\left(x^{\alpha}\right)\right) \\
& =x^{\alpha}-(\lambda w+x) \\
& =\left(\frac{\alpha}{\sum_{k=1}^{n} w_{k}^{2}}\left(\gamma_{1}-\gamma_{0}\right)-\lambda\right) w .
\end{aligned}
$$

This implies that $q\left(x^{\alpha}\right)-p\left(q\left(x^{\alpha}\right)\right)=\delta w$ for some $\delta \in \mathbb{R}$. Since $q\left(x^{\alpha}\right) \in \operatorname{bnd}(Q),\left\|q\left(x^{\alpha}\right)-p\left(q\left(x^{\alpha}\right)\right)\right\|_{2}=1$, so $\delta \in\left\{-\frac{1}{\|w\|_{2}}\right.$, $\left.\frac{1}{\|w\|_{2}}\right\}$, which in turn implies that $q\left(x^{\alpha}\right) \in Q^{0} \cup Q^{1}$, as only points $\bar{q}$ in $Q^{0} \cup Q^{1}$ satisfy that $\bar{q}-p(\bar{q})$ is a strictly positive multiple of 
w. Suppose $q\left(x^{\alpha}\right) \in Q^{0}$. Now $x^{\alpha} \in X^{\alpha}$ and $q\left(x^{\alpha}\right) \in Q^{0}$ implies $x^{\alpha}=q\left(x^{\alpha}\right)$, so $x^{\alpha} \in Q^{0}$. A similar argument shows that $q\left(x^{\alpha}\right) \in Q^{1}$ implies $x^{\alpha} \in Q^{1}$. In both case we obtain a contradiction to $x^{\alpha} \notin Q$. Consequently, $x^{\alpha} \in Q$.

Next, suppose that $x^{\alpha} \in \operatorname{int}(Q)$. Then $x^{\alpha}-\alpha / \sum_{k=1}^{n} w_{k}^{2}\left(\gamma_{1}-\gamma_{0}\right) w=x=\tau\left(x^{\alpha}+z\left(x^{\alpha}\right)\right)$. Since $\tau\left(x^{\alpha}+z\left(x^{\alpha}\right)\right)=x^{\alpha}+z\left(x^{\alpha}\right)-\lambda w$ for some $\lambda \in \mathbb{R}$, it follows that

$$
z\left(x^{\alpha}\right)=\left(\lambda-\frac{\alpha}{\sum_{k=1}^{n} w_{k}^{2}}\left(\gamma_{1}-\gamma_{0}\right)\right) w .
$$

Because of Walras' law, we obtain

$$
p\left(x^{\alpha}\right) \cdot z\left(x^{\alpha}\right)=\left(\lambda-\frac{\alpha}{\sum_{k=1}^{n} w_{k}^{2}}\left(\gamma_{1}-\gamma_{0}\right)\right) p\left(x^{\alpha}\right) \cdot w=0 .
$$

Hence, since $p\left(x^{\alpha}\right) \cdot w>0$, we have that $\lambda=\alpha / \sum_{k=1}^{n} w_{k}^{2}\left(\gamma_{1}-\gamma_{0}\right)$ and thus $z\left(x^{\alpha}\right)=0^{n}$, showing that $x^{\alpha}$ is a zero point of $z$.

Finally, suppose $x^{\alpha} \in \operatorname{bnd}(Q)$. Then there exists $\beta_{1} \geq 0$ and $\beta_{2} \geq 0$ with $\beta_{1}+\beta_{2}=1$ such that

$$
\beta_{1} \tau\left(x^{\alpha}+z\left(x^{\alpha}\right)\right)+\beta_{2} \tau\left(p\left(x^{\alpha}\right)\right)=x=x^{\alpha}-\frac{\alpha}{\sum_{k=1}^{n} w_{k}^{2}}\left(\gamma_{1}-\gamma_{0}\right) w .
$$

Using that $\tau(\bar{x})$ is the projection of $\bar{x}$ on $X^{0}$, it follows that this can be rewritten as

$$
\beta_{1} x^{\alpha}+\beta_{1} z\left(x^{\alpha}\right)+\beta_{2} p\left(x^{\alpha}\right)-\mu w=x^{\alpha}-\frac{\alpha}{\sum_{k=1}^{n} w_{k}^{2}}\left(\gamma_{1}-\gamma_{0}\right) w,
$$

for some $\mu \in \mathbb{R}$. With $\beta_{1}+\beta_{2}=1$ and letting $\lambda=\mu-\frac{\alpha}{\sum_{k=1}^{n} w_{k}^{2}}\left(\gamma_{1}-\gamma_{0}\right)$ we obtain

$$
\beta_{1} z\left(x^{\alpha}\right)=\beta_{2}\left(x^{\alpha}-p\left(x^{\alpha}\right)\right)+\lambda w .
$$

First, suppose $\beta_{1}=0$. Then, as before, it follows that $x^{\alpha}-p\left(x^{\alpha}\right)=\delta w$ for some $\delta \in\left\{-1 /\|w\|_{2}, 1 /\|w\|_{2}\right\}$, implying that $x^{\alpha} \in Q^{0} \cup Q^{1}$. Hence, according to Theorem $4.7, z\left(x^{\alpha}\right)=0^{n}$ and thus $x^{\alpha}$ is a zero point of $z$. Second, suppose $\beta_{1}>0$. Then

$$
z\left(x^{\alpha}\right)=\frac{\beta_{2}}{\beta_{1}}\left(x^{\alpha}-p\left(x^{\alpha}\right)\right)+\frac{\lambda}{\beta_{1}} w=\frac{\delta}{\beta_{1}} r\left(x^{\alpha}\right)+\frac{\lambda}{\beta_{1}} w,
$$

for some $\delta \geq 0$. Since $x^{\alpha} \in \operatorname{bnd}(Q)$, we have that there is $k$ such that $\left|r_{k}\left(x^{\alpha}\right)\right|=w_{k}$. When $r_{k}\left(x^{\alpha}\right)=-w_{k}$ it follows that

$$
z_{k}\left(x^{\alpha}\right)=-\frac{\delta}{\beta_{1}} w_{k}+\frac{\lambda}{\beta_{1}} w_{k} \geq 0,
$$

so $\lambda \geq \delta$. Hence, $-\delta+\lambda \geq 0$ and thus

$$
z_{h}\left(x^{\alpha}\right)=\frac{\delta}{\beta_{1}} r_{h}\left(x^{\alpha}\right)+\frac{\lambda}{\beta_{1}} w_{h} \geq-\frac{\delta}{\beta_{1}} w_{h}+\frac{\lambda}{\beta_{1}} w_{h} \geq 0,
$$

for all $h \in I_{n}$ and therefore $z\left(x^{\alpha}\right)=0^{n}$ due to Walras' law. Similarly, when $r_{k}\left(x^{\alpha}\right)=w_{k}$ it follows that

$$
z_{k}\left(x^{\alpha}\right)=\frac{\delta}{\beta_{1}} w_{k}+\frac{\lambda}{\beta_{1}} w_{k} \leq 0,
$$

for some $\delta \geq 0$ and thus $\delta+\lambda \leq 0$. Then

$$
z_{h}\left(x^{\alpha}\right)=\frac{\delta}{\beta_{1}} r_{h}\left(x^{\alpha}\right)+\frac{\lambda}{\beta_{1}} w_{h} \leq \frac{\delta}{\beta_{1}} w_{h}+\frac{\lambda}{\beta_{1}} w_{h} \leq 0,
$$

for all $h \in I_{n}$ and therefore $z\left(x^{\alpha}\right)=0^{n}$ due to Walras' law. 
The theorem says that there is a connected set $C$ in $Q$ of zero points of the reduced excess demand function $z$, containing at least a trivial zero point $q^{0}$ in $Q^{0}$ and a trivial zero point $q^{1}$ in $Q^{1}$. Since $Q^{0}$ and $Q^{1}$ have an empty intersection, there must be a connected set of non-trivial zero points between $Q^{0}$ and $Q^{1}$. According to Lemma 4.6 every non-trivial zero point $q^{*} \in C$ of $z$ induces a non-trivial quantity constrained equilibrium $\left(p^{*}, r^{*}, x^{*}\right)=\left(p\left(q^{*}\right), r\left(q^{*}\right), d\left(q^{*}\right)\right)$, where $d\left(q^{*}\right)=\left(d^{1}\left(q^{*}\right), \ldots, d^{m}\left(q^{*}\right)\right)$ is the demand allocation induced by $q^{*}$. In order to prove Theorem 4.2 we still have to show that the set $\{(p(q), r(q), d(q)) \mid q \in C\}$ is a connected set.

Proof of Theorem 4.2. Take the function $f: Q \rightarrow P \times \mathbb{R}^{n} \times \prod_{i \in I_{m}} \mathcal{X}^{i}$ defined by

$$
f(q)=\left(p(q), r(q), d^{1}(q), \ldots, d^{m}(q)\right), \quad q \in Q .
$$

Consider the connected set $C$ of zero points of $z$ as defined in Theorem 4.8. It follows by Lemma 4.6 that every point in $C$ induces a QCE of $\mathcal{E}$. Since $f$ is a continuous function and by Theorem $4.8 C$ is connected, it follows that the set $D=\{x \mid x=$ $f(q), q \in C$ \} is connected. By Lemma 4.7, it follows that $f(q)$ is a fully supply-constrained equilibrium whenever $q \in Q^{0}$ and $f(q)$ is a fully demand-constrained equilibrium whenever $q \in Q^{1}$. Since $C$ intersects both $Q^{0}$ and $Q^{1}$, the set $D$ contains both a fully supply-constrained equilibrium and a fully demand-constrained equilibrium.

We have proved now that there exists a connected set of non-trivial quantity constrained equilibria connecting a no-trade equilibrium with completely pessimistic expectations concerning supply opportunities and a no-trade equilibrium with completely pessimistic expectations concerning demand opportunities. Each quantity constrained equilibrium is induced by some $q \in Q$. Any $q$ in $Q^{0}$ or in $Q^{1}$ yields a trivial quantity constrained equilibrium, whereas any $q^{*}$ in $C$ not in $Q^{0}$ or $Q^{1}$ yields a non-trivial quantity constrained equilibrium. If $q^{*} \in P$, then $p\left(q^{*}\right)=q^{*}$ is a Walrasian equilibrium price vector, the rationing vector $r\left(q^{*}\right)=0^{n}$, and the induced expected trade opportunities given by $\left(\ell^{*}, u^{*}\right)=(-w, w)$ are not binding, i.e. no consumer faces binding expected trade opportunities on either the demand side or the supply side and there is no coordination failure. If $q^{*}$ is not in $P$ and so $p\left(q^{*}\right) \neq q^{*}$, then the price vector $p^{*}=p\left(q^{*}\right)$ lies on the boundary of the set $P$ of admissible prices and the rationing vector $r^{*}=r\left(q^{*}\right)$ lies in the normal cone $G\left(p^{*}\right)$ at $p^{*}$ to $P$, and so the prices cannot be adjusted further at $p^{*}$ in the direction $r^{*}$. The vector $r^{*}$ then determines the expected trade opportunities $\left(\ell^{*}, u^{*}\right)=\left(-w-r^{*}, w-r^{*}\right)$. Expected trade opportunities on supply can only be binding for commodity $k$ when $r_{k}^{*}<0$ and expected opportunities on demand can only be binding for commodity $h$ when $r_{h}^{*}>0$.

Having proved that there exists a connected set of QCEs connecting a trivial fully supply-constrained no-trade equilibrium and a trivial fully demand-constrained no-trade equilibrium, it is easy to show that this set contains several non-trivial QCEs with some specific properties. These properties are most easily stated in terms of the parametrization by the set $Q$, with each $q \in Q$ inducing a price $p(q)$, a rationing vector $r(q)$, and an allocation $\left(d^{1}(q), \ldots, d^{m}(q)\right)$.

First of all, recall that for any $q^{0} \in C \cap Q^{0}$ it holds that $r\left(q^{0}\right)=-w$ and that for any $q^{1} \in C \cap Q^{1}$ it holds that $r\left(q^{1}\right)=w$. Therefore, since $C$ connects $Q^{0}$ and $Q^{1}$ and $r$ is a continuous function on $Q$ for any a priori chosen $k \in I_{n}$ there exists a $q(k) \in C$ satisfying $r_{k}(q(k))=0$. Such a $q(k)$ induces expected trade opportunities $(\ell, u)$ with $\ell_{k}=-w_{k}$ and $u_{k}=w_{k}$. The point $q(k)$ in $Q$ therefore induces a QCE at which no consumer faces binding expected opportunities concerning commodity $k$. We call such an equilibrium a generalized Drèze equilibrium with respect to commodity $k$.

Corollary 4.9. For any $k \in I_{n}$, there exists a generalized Drèze equilibrium with respect to commodity $k$.

The corollary implies that if commodity $k$ is the numeraire, there exists a QCE at which there are no binding expected trade opportunities concerning the numeraire.

The connected set $C$ also contains a point $q^{-}$satisfying $\max _{j} r_{j}\left(q^{-}\right)=0$. This element of $C$ induces a QCE where for at least one commodity there are no binding expected trade opportunities and for all other commodities there may only be binding expected supply opportunities and therefore is a generalized supply-constrained equilibrium. Similarly, there exists a QCE at which consumers may only face binding expected demand opportunities and for at least one commodity there are no binding expected trade opportunities, and is therefore what we call a generalized demand-constrained equilibrium. Such an equilibrium is induced by a point $q^{+}$in $C$ for which $\min _{j} r_{j}\left(q^{+}\right)=0$. Notice that both types of equilibria are QCEs for which there are no binding expected trade opportunities concerning at least one commodity, but ex ante it cannot be said which commodity.

Corollary 4.10. There exists a generalized supply-constrained equilibrium and there exists a generalized demand-constrained equilibrium.

Since we have not made any differentiability assumptions, we cannot say more about the structure of the set of equilibria than the statement in Theorem 4.2 that there is a continuum connecting two extreme equilibria, i.e. loosely speaking the dimension of the set of equilibria is at least one. We argue next that in general one should expect the dimension to be exactly one. We can parametrize prices and the rationing vector $r$ by means of an $n$-dimensional vector $q$. Using Lemma 4.6, we can show that the set of equilibria corresponds to the set of $q$ 's solving $z(q)=0^{n}$. The system $z(q)=0^{n}$ consists of $n-1$ independent equations by virtue of Walras' law as stated in Lemma 4.5. This leaves one degree of freedom, resulting in a one-dimensional set of equilibria.

From Theorem 4.2 it also follows that for every $v \in[\min w \cdot P, \max w \cdot P]$ there exists a quantity constrained equilibrium $\left(p^{*}, r^{*}, x^{*}\right)$ satisfying $\sum_{k=1}^{n} p_{k}^{*} w_{k}=v$. In case for $P$ it holds that $\min w \cdot P=\max w \cdot P$ every QCE $\left(p^{*}, r^{*}, x^{*}\right)$ has the property 
that $\sum_{k=1}^{n} p_{k}^{*} w_{k}=v$ for the same $v$. In case $\min w \cdot P<\max w \cdot P$ and we take some $v, \min w \cdot P<v<\max w \cdot P$, there is one degree of freedom less and one should typically expect a finite number of QCEs $\left(p^{*}, r^{*}, x^{*}\right)$ satisfying $\sum_{k=1}^{n} p_{k}^{*} w_{k}=v$.

\section{Applications and extensions}

\subsection{Price indexation}

In this subsection we apply the concept of QCE as defined in Definition 3.1 to the set of admissible prices given by

$$
P=\left\{p \in \mathbb{R}_{+}^{n} \mid \underline{p}_{j} \leq p_{j} \leq \bar{p}_{j}, j \in I, \underline{\pi}_{j}\left(p_{I}\right) \leq p_{j} \leq \bar{\pi}_{j}\left(p_{I}\right), j \in J\right\},
$$

where $I$ and $J$ partition $I_{n}$. This set satisfies the so-called noncircularity condition of Weddepohl (1987), saying that the prices may not be indexed directly or indirectly by themselves. Dehez and Drèze (1984) and van der Laan (1984) have considered special cases of this set by making additional assumptions on the upper and lower bound of the index commodities or on the index functions, see also Chetty and Nayak (1978), Kurz (1982) and Weddepohl (1987). Here we only make some weak assumptions by assuming that for any $j \in I$ it holds that $0<\underline{p}_{j} \leq \bar{p}_{j}<\infty$ and that for any $j \in J$, the lower bound function $\underline{\pi}_{j}$ is convex, the upper bound function $\bar{\pi}_{j}$ is concave, and that for all feasible $p_{I}$ it holds that $0<\pi_{j}\left(p_{I}\right) \leq \bar{\pi}_{j}\left(p_{I}\right)<\infty$, so that $P$ satisfies Assumption P. To give a characterization of a QCE, for simplicity we assume that all index functions are continuously differentiable.

Suppose $q \in Q$ induces a QCE and thus $z(q)=0^{n}$. Since $r(q) \in G(p(q))$, we have that $p(q) \cdot r(q)=\max _{p \in P} p \cdot r(q)$. From the first-order Kuhn-Tucker conditions it then follows that there exists nonnegative numbers $\underline{\lambda}_{j}$ and $\bar{\lambda}_{j}, j \in I_{n}$, such that

$$
\begin{aligned}
& r_{j}(q)=\bar{\lambda}_{j}-\underline{\lambda}_{j}+\sum_{h \in J} \underline{\lambda}_{h} \frac{\partial \underline{\pi}_{h}\left(p_{I}(q)\right)}{\partial p_{j}}-\sum_{h \in J} \bar{\lambda}_{h} \frac{\partial \bar{\pi}_{h}\left(p_{I}(q)\right)}{\partial p_{j}}, j \in I, \\
& r_{j}(q)=\bar{\lambda}_{j}-\underline{\lambda}_{j}, j \in J
\end{aligned}
$$

and

$$
\begin{aligned}
& \underline{\lambda}_{j}\left(p_{j}(q)-\underline{p}_{j}\right)=0, \bar{\lambda}_{j}\left(\bar{p}_{j}-p_{j}(q)\right)=0, \underline{\lambda}_{j} \bar{\lambda}_{j}=0, j \in I, \\
& \underline{\lambda}_{j}\left(p_{j}(q)-\underline{\pi}_{j}\left(p_{I}(q)\right)\right)=0, \bar{\lambda}_{j}\left(\bar{\pi}_{j}\left(p_{I}(q)\right)-p_{j}(q)\right)=0, \underline{\lambda}_{j} \bar{\lambda}_{j}=0, j \in J .
\end{aligned}
$$

Recall that in a QCE Condition (iii) of Definition 3.2 is satisfied, but not necessarily Condition (iv) of Definition 2.1. So, when there are binding expected supply (demand) opportunities concerning a commodity, its price is not necessarily on its lower (upper) bound. To make this more precise, take an indexed commodity $j \in J$. From (3) and the last complementarity condition in (5) it follows that

$$
\underline{\lambda}_{j}=-r_{j}(q)>0 \quad \text { if } r_{j}(q)<0, \quad \text { and } \quad \bar{\lambda}_{j}=r_{j}(q)>0 \quad \text { if } r_{j}(q)>0 .
$$

From the first two complementarity conditions in (5) it then follows that $p_{j}(q)=\underline{\pi}_{j}\left(p_{I}(q)\right)$ if there are binding expected supply opportunities for commodity $j \in J$ and that $p_{j}(q)=\bar{\pi}_{j}\left(p_{I}(q)\right)$ if there are binding demand opportunities for commodity $j \in J$, i.e. in case of binding expected supply (demand) opportunities the price of an indexed commodity is on its lower (upper) bound given the prices $p_{I}(q)$ of the index commodities. For an index commodity $j \in I$, in general such a clear link does not exist. For instance, suppose that all index functions are monotonically increasing in $p_{I}$ and all the derivatives are positive. Now, if $p_{k}(q)<\bar{\pi}_{k}\left(p_{I}(q)\right)$ for all $k \in J$, then $\bar{\lambda}_{k}=0$ for all $k \in J$ and it follows from Eq. (2) that for all $j \in I$ it holds

$$
r_{j}(q)=\bar{\lambda}_{j}-\underline{\lambda}_{j}+\sum_{k \in J} \underline{\lambda}_{k} \frac{\partial \underline{\pi}_{k}\left(p_{I}(q)\right)}{\partial p_{j}}
$$

If also $p_{k}(q)>\underline{\pi}_{k}\left(p_{I}(q)\right)$ for all $k \in J$, then $\underline{\lambda}_{k}=0$ for all $k \in J$ and thus $r_{j}(q)=\bar{\lambda}_{j}-\underline{\lambda}_{j}$ for all $j \in I$, implying that there can only be binding expected trade opportunities for an index commodity $j \in I$ when its price is on its upper or lower bound. However, when the price of some indexed commodity is on its lower bound, then $\sum_{k \in J} \underline{\lambda}_{k}\left(\partial \underline{\pi}_{k}\left(p_{I}(q)\right) / \partial p_{j}\right)$ is positive and thus $r_{j}(q)$ may be positive even if $\bar{\lambda}_{j}=0$ and thus $p_{j}(q)$ is not on its upper bound. In such a situation binding expected demand opportunities of an index commodity may occur, not because its price is on its upper bound, but caused by the fact that the prices of the indexed commodities cannot be decreased further. Similarly, if the price of some indexed commodity is on its upper bound, there may be binding expected supply opportunities on index commodities, even when the prices of these index commodities are not on their lower bounds.

Corollary 5.1. Let $P$ be given as in (1) and let $q \in Q$ be such that $z(q)=0^{n}$. Then, for all $j \in J$,

$$
p_{j}(q)=\underline{\pi}_{j}\left(p_{I}(q)\right) \quad \text { ifr } r_{j}(q)<0 \quad \text { and } \quad p_{j}(q)=\bar{\pi}_{j}\left(p_{I}(q)\right) \quad \text { ifr } r_{j}(q)>0 .
$$


When all index functions are monotonically increasing, then, for all $j \in I$,

$$
p_{j}(q)=\underline{p}_{j} \quad \text { if } r_{j}(q)<0 \quad \text { and } \quad \text { for all } k \in J, p_{k}(q)<\bar{\pi}_{k}\left(p_{I}(q)\right),
$$

and

$$
p_{j}(q)=\bar{p}_{j} \quad \text { if } r_{j}(q)>0 \quad \text { and } \quad \text { for all } k \in J, p_{k}(q)>\underline{\pi}_{k}\left(p_{I}(q)\right) .
$$

Moreover, for $j \in I$,

$$
p_{j}(q)>\underline{p}_{j} \text { and } r_{j}(q)<0 \text { implies that } p_{k}(q)=\bar{\pi}_{k}\left(p_{I}(q)\right) \text {, for at least one } k \in J \text {, }
$$

and

$$
p_{j}(q)<\bar{p}_{j} \text { and } r_{j}(q)>0 \text { implies that } p_{k}(q)=\underline{\pi}_{k}\left(p_{I}(q)\right) \text {, for at least one } k \in J \text {. }
$$

Corollary 5.1 characterizes a QCE in case the set $P$ of admissible prices is given as in (1) and generalizes therefore the results obtained in the literature before. In particular, for every $k \in I_{n}$, there exists a generalized Drèze equilibrium with respect to commodity $k$ and there exists both a generalized supply-constrained and a generalized demand-constrained equilibrium.

\subsection{Unbounded sets of admissible prices}

In this subsection we consider an extension allowing that the set of admissible prices is unbounded. We show that there is an unbounded connected set of QCEs with at least one of the prices going to infinity. Let $P$ be a convex, closed and unbounded set of admissible prices in the interior of $\mathbb{R}_{+}^{n}$ and define $Q=\left\{q \in \mathbb{R}^{n} \mid\|q-p\|_{2} \leq 1\right.$, for some $\left.p \in P\right\}$. Notice that the set $Q^{0}$ as defined before is non-empty, while the set $Q^{1}$ is empty. The next theorem says that there exists an unbounded connected set of zero points of $z$ in $Q$ containing a point in $Q^{0}$. Again, a zero point of $z$ in $Q^{0}$ induces a fully supply-constrained no-trade equilibrium.

Theorem 5.2. Let $\mathcal{E}=\left(\left\{\mathcal{X}^{i}, \succcurlyeq^{i}, w^{i}\right\}_{i=1}^{m}, P\right)$ be an economy satisfying Assumptions $\mathrm{X}, \mathrm{U}$ and $\mathrm{W}$, and let $P$ be a non-empty, convex, closed, unbounded set in the interior of $\mathbb{R}_{+}^{n}$. Then there exists an unbounded connected subset $C$ of $Q$ such that $C \cap Q^{0} \neq \emptyset$ and each point in $C$ is a zero point of the reduced excess demand function $z$.

Proof. Let $\gamma_{0}=\sum_{k=1}^{n} w_{k} q_{k}$ for any $q \in Q^{0}$, and for $\gamma>\gamma_{0}$ define

$$
\begin{aligned}
& P(\gamma)=\left\{p \in P \mid \sum_{k=1}^{n} w_{k} p_{k} \leq \gamma\right\}, \\
& Q(\gamma)=\left\{q \in Q \mid \sum_{k=1}^{n} w_{k} q_{k} \leq \gamma\right\}, \\
& \bar{Q}(\gamma)=\left\{q \in Q \mid \sum_{k=1}^{n} w_{k} q_{k}=\gamma\right\} .
\end{aligned}
$$

For $q \in Q$, the vectors $p(q), r(q)$ and $z(q)$ are defined as before. Let $\bar{\gamma}$ be a number satisfying $\bar{\gamma}>\gamma+\|w\|_{2}$. Then the set of prices $P(\bar{\gamma})$ satisfies the assumptions of Theorem 4.8. Let $Q_{\bar{\gamma}}, Q_{\bar{\gamma}}^{0}$ and $Q_{\bar{\gamma}}^{1}$ be the corresponding sets (i.e. as the sets $Q, Q^{0}$ and $Q^{1}$ corresponding to $P$ defined in Section 4 ) and let $p_{\bar{\gamma}}, r_{\bar{\gamma}}$ and $z_{\bar{\gamma}}$ be the corresponding functions on $Q_{\bar{\gamma}}$ (i.e. as the functions $p, r$ and $z$ defined on $Q$ in Section 4$)$. Applying Theorem 4.8 there is a connected set $C_{\bar{\gamma}}$ of zero points in $Q_{\bar{\gamma}}$ that intersects $Q_{\bar{\gamma}}^{0}$ and $Q_{\bar{\gamma}}^{1}$. Clearly, $C_{\bar{\gamma}} \cap Q(\gamma)$ contains a connected subset $C(\gamma)$ that intersects $Q_{\bar{\gamma}}^{0}$ and $\bar{Q}(\gamma)$. Since $\bar{\gamma}>\gamma+\|w\|_{2}$, we have that $Q(\gamma)=Q_{\bar{\gamma}} \cap Q(\gamma)$ and that the restrictions of the functions $p, r$ and $z$ to $Q(\gamma)$ coincide with the restrictions of the functions $p_{\bar{\gamma}}, r_{\bar{\gamma}}$ and $z_{\bar{\gamma}}$ to $Q_{\bar{\gamma}} \cap Q(\gamma)$. The set $C(\gamma)$ is therefore a connected set of zero points of the function $z$ in $Q(\gamma)$ containing a point in $Q^{0}$ and a point in $\bar{Q}(\gamma)$. Finally, take $C=Q^{0} \cup\left(\cup_{\gamma>\gamma_{0}} C(\gamma)\right)$. Since any point in $Q^{0}$ is a zero point of $z$, it follows that the set $C$ is an unbounded connected set of zero points of $z$ in $Q$ such that $C \cap Q^{0} \neq \emptyset$.

As a specific example we consider, for some arbitrarily small $\varepsilon>0$, the set $P$ given by

$$
P=\left\{p \in \mathbb{R}_{+}^{n} \mid p_{j} \geq \varepsilon, j \in M, \sum_{j \in I} p_{j}=1, \underline{p}_{j} \leq p_{j} \leq \bar{p}_{j}, j \in I \cup J\right\},
$$

where $M, I$ and $J$ form a partition of $I_{n}{ }^{2}$ The set $M$ is referred to as the set of numeraire commodities. For $M=\emptyset$ and $\underline{p}_{j}=0, \bar{p}_{j}=$ $\infty$ for $j \in I$, this set has been considered by van der Laan (1984) as a special case of the set given in formula (1). Here we assume

\footnotetext{
${ }^{2}$ For simplicity we take prices bounded away from zero. The analysis can be extended to $\varepsilon=0$ by generalizing the concept of constrained equilibrium to quasi-constrained equilibrium as has been discussed in Kurz (1982).
} 
that for any $j \in I \cup J$ it holds that $0<\underline{p}_{j} \leq \bar{p}_{j}<\infty$ and $\sum_{j \in I} \underline{p}_{j}<1<\sum_{j \in I} \bar{p}_{j}$. Without loss of generality, we may assume that $I=\{1, \ldots,|I|\}$ and $w_{j+1} \geq w_{j}, j=1, \ldots|I|-1$. Moreover, for the sake of simplicity, we restrict ourselves to the generic case that there is no partition $I^{1}, I^{2}$ of $I$ such that $\sum_{j \in I^{1}} \underline{p}_{j}+\sum_{j \in I^{2}} \bar{p}_{j}=1$. Then, let $j^{\prime} \in I$ be such that $\sum_{j=1}^{j^{\prime}} \underline{p}_{j}+\sum_{j=j^{\prime}+1}^{|I|} \bar{p}_{j}<1$ and $\sum_{j=1}^{j^{\prime}-1} \underline{p}_{j}+\sum_{j=j^{\prime}}^{|I|} \bar{p}_{j}>1$.

Suppose $q \in Q$ induces a QCE and thus $z(q)=0^{n}$. Again, $r(q) \in G(p(q))$ implies $p(q) \cdot r(q)=\max _{p \in P} p \cdot r(q)$. From the first-order Kuhn-Tucker conditions it then follows that there exist nonnegative numbers $\lambda_{j}, j \in M$, nonnegative numbers $\underline{\lambda}_{j}, \bar{\lambda}_{j}, j \in I \cup J$, and a number $\lambda_{I}$ such that

$$
\begin{aligned}
& r_{j}(q)=-\lambda_{j}, j \in M, \\
& r_{j}(q)=\lambda_{I}-\underline{\lambda}_{j}+\bar{\lambda}_{j}, j \in I, \\
& r_{j}(q)=-\underline{\lambda}_{j}+\bar{\lambda}_{j}, j \in J,
\end{aligned}
$$

and furthermore the following complementarity conditions hold

$$
\begin{aligned}
& \lambda_{j}\left(p_{j}(q)-\varepsilon\right)=0, j \in M, \\
& \underline{\lambda}_{j}\left(p_{j}(q)-\underline{p}_{j}\right)=0, \bar{\lambda}_{j}\left(\bar{p}_{j}-p_{j}(q)\right)=0, \underline{\lambda}_{j} \bar{\lambda}_{j}=0, j \in I \cup J .
\end{aligned}
$$

At a fully supply-constrained no-trade equilibrium we have that $r(q)=-w$, so that $\lambda_{j}=w_{j}$ and hence $p_{j}(q)=\varepsilon$ for all $j \in M, \underline{\lambda}_{j}=w_{j}, \bar{\lambda}_{j}=0$ and hence $p_{j}(q)=\underline{p}_{j}$ for all $j \in J$, while for $j \in I$ it holds

$$
\lambda_{I}-\underline{\lambda}_{j}+\bar{\lambda}_{j}=-w_{j}
$$

We claim that $\lambda_{I}=-w_{j^{\prime}}$. For all $j=1, \ldots, j^{\prime}-1$ it holds that $\underline{\lambda}_{j}=0$ and $\bar{\lambda}_{j}=w_{j^{\prime}}-w_{j} \geq 0, \underline{\lambda}_{j^{\prime}}=\bar{\lambda}_{j^{\prime}}=0$, and for all $j=$ $j^{\prime}+1, \ldots,|I|$ it holds that $\bar{\lambda}_{j}=0$ and $\underline{\lambda}_{j}=w_{j}-w_{j^{\prime}} \geq 0$. It then follows immediately that prices of commodities $j=1, \ldots, j^{\prime}-1$ satisfy $p_{j}=\bar{p}_{j}$, and prices of commodities $j=j^{\prime}+1, \ldots,|I|$ satisfy $p_{j}=\underline{p}_{j}$. Intuitively, from the properties that $p(q) \cdot r(q)=$ $\max _{p \in P} p \cdot r(q), r(q)=-w$ and $\sum_{j \in I} p_{j}=1$, it follows that the prices of the commodities with the larger total endowments are set on their minimum level, and the prices of commodities with the smaller total endowments on their maximum level.

To formally show our claim, consider a commodity $k \in\{1, \ldots,|I|\}=I$ such that $\underline{p}_{k}<p_{k}(q)<\bar{p}_{k}$. Such a commodity $k$ exists, since we consider the case that there is no partition $I^{1}, I^{2}$ of $I$ such that $\sum_{j \in I^{1}} \underline{p}_{j}+\sum_{j \in I^{2}} \bar{p}_{j}=1$. It follows immediately that $\underline{\lambda}_{k}=\bar{\lambda}_{k}=0$, so $\lambda_{I}=-w_{k}$. Consider any commodity $j \in\{1, \ldots, k-1\}$. Then it follows from Eq. (8) that $\underline{\lambda}_{j}-\bar{\lambda}_{j}=\lambda_{I}-r_{j}(q)=$ $\lambda_{I}+w_{j}=-w_{k}+w_{j} \leq 0$, so that $\underline{\lambda}_{j}=0$ and $\bar{\lambda}_{j}=w_{k}-w_{j} \geq 0$. Similarly, for any commodity $j \in\{k+1, \ldots,|I|\}$ it follows from Eq. (8) that $\underline{\lambda}_{j}-\bar{\lambda}_{j}=-w_{k}+w_{j} \geq 0$, so that $\bar{\lambda}_{j}=0$ and $\underline{\lambda}_{j}=-w_{k}+w_{j} \geq 0$. Together with the complementarity properties given in (11) it follows that

$$
\sum_{j=1}^{k-1} p_{j}(q)+p_{k}(q)+\sum_{j=k+1}^{|I|} p_{j}(q)=\sum_{j=1}^{k-1} \bar{p}_{j}+p_{k}(q)+\sum_{j=k+1}^{|I|} \underline{p}_{j}=1 .
$$

Therefore,

$$
\begin{aligned}
& \sum_{j=1}^{k} \bar{p}_{j}+\sum_{j=k+1}^{|I|} \underline{p}_{j} \geq 1 \\
& \sum_{j=1}^{k-1} \bar{p}_{j}+\sum_{j=k}^{|I|} \underline{p}_{j} \leq 1
\end{aligned}
$$

so $k=j^{\prime}$. Summarizing, at a fully supply-constrained equilibrium, the prices of all the numeraire and indexed commodities are on its lower bound, while the price of precisely one index commodity is not bounded and the prices of the index commodities with larger total endowments are set on their minimum level, and the prices of the index commodities with smaller total endowments on their maximum level.

From Eqs. (7)-(11) it follows that at any QCE it holds that there may be binding expected supply opportunities for a numeraire commodity, which only occurs when its price is $\varepsilon$, while there can only be binding expected supply (demand) opportunities for an indexed commodity when its price is on its lower (upper) bound. For an index commodity $j \in I$ binding expected opportunities can also occur when its price is not binding, due to the restriction that the sum of the prices of the index commodities is equal to one. For all commodities $j \in I$ with non-binding prices we have that $r_{j}(q)=\lambda_{I}$. Therefore, for all these commodities there are either no binding expected trade opportunities $\left(\lambda_{I}=0\right)$, or the same amount of expected trade opportunities on either their demands $\left(\lambda_{I}>0\right)$ or their supplies $\left(\lambda_{I}<0\right)$. At a QCE induced by a point $q$ satisfying $\sum_{j \in I} q_{j}=1$, it holds that $\lambda_{I}=0$ and there can only be binding expected supply (demand) opportunities for a commodity in 
$I$ if its price is on its lower (upper) bound. This confirms the results in van der Laan (1984) for the case that $M=\emptyset$. When $M \neq \emptyset$, the following result can also be obtained.

Theorem 5.3. Let $\mathcal{E}=\left(\left\{\mathcal{X}^{i}, \succcurlyeq^{i}, w^{i}\right\}_{i=1}^{m}, P\right)$ be an economy satisfying Assumptions $\mathrm{X}, \mathrm{U}$ and $\mathrm{W}$, and let the set $P$ be as specified in (6). Then there exists $\bar{\gamma}>\gamma_{0}$ such that for all zero points $q \in Q$ of $z$ with $\sum_{k=1}^{n} w_{k} q_{k} \geq \bar{\gamma}$ it holds that $r_{j}(q)=w_{j}$ for all $j \in I \cup J, p_{j}(q)>\varepsilon$ and $r_{j}(q)=0$ for all $j \in M$.

Proof. From Theorem 5.2 it follows that there exists a sequence $\left(q^{t}\right)_{t \in N}$ in $Q$, satisfying $\sum_{h=1}^{n} w_{h} q_{h}^{t} \geq t$ and $z\left(q^{t}\right)=0^{n}$ for all $t \in \mathrm{IN}$. Since $P$ and thus also $Q$ is bounded in any $j \in I \cup J$, without loss of generality it follows that $q_{k}^{t} \rightarrow \infty$ and therefore $p_{k}^{t}(q) \rightarrow$ $\infty$ for some $k \in M$. It is obvious that for this $k$ it holds that $r_{k}\left(q^{t}\right)=0$. Now, define $I^{t}=\left\{j \in I \mid r_{j}\left(q^{t}\right)<w_{j}\right\}, J^{t}=\left\{j \in J \mid r_{j}\left(q^{t}\right)<w_{j}\right\}$ and $M^{t}=\left\{j \in M \mid p_{j}\left(q^{t}\right)=\varepsilon\right\}$. Then, for any $j \in I \cup J \cup M^{t}$ we have that $p_{j}\left(q^{t}\right) / p_{k}\left(q^{t}\right)$ goes to zero. Moreover, for all $i \in I_{m}$ and $j \in I_{n}$, the demand $d_{j}^{i}\left(q^{t}\right)$ is bounded from above by $w_{j}^{i}+\left(w_{j}-r_{j}\left(q^{t}\right)\right)$, while $r_{k}\left(q^{t}\right)=0$ implies net supply equal to $-w_{k}$ is possible for commodity $k$. For $t$ sufficiently large, for any $i \in I_{m}$, and for any $j \in I^{t} \cup J^{t} \cup M^{t}$, it follows from the monotonicity of the preferences that $d_{j}^{i}\left(q^{t}\right)=w_{j}^{i}+\left(w_{j}-r_{j}\left(q^{t}\right)\right)>w_{j}^{i}$, contradicting $z\left(q^{t}\right)=0^{n}$. Hence $I^{t} \cup J^{t} \cup M^{t}=\emptyset$. So, for $t$ sufficiently large it follows that $r_{j}\left(q^{t}\right)=w_{j}$ for all $j \in I \cup J$, and $p_{j}\left(q^{t}\right)>\varepsilon$ and $r_{j}\left(q^{t}\right)=0$ for all $j \in M$.

The theorem implies that there exists a connected set of QCEs containing a fully supply-constrained equilibrium and an equilibrium with completely pessimistic expectations concerning the demand of the commodities in $I \cup J$ and no binding expected trade opportunities at all on the commodities in $M$ with prices above $\varepsilon$. Clearly, at the latter equilibrium it must hold that for every consumer the consumption of the commodities in $I \cup J$ is equal to its initial endowments of these commodities. Hence the prices of the commodities in $M$ are Walrasian equilibrium prices for a reduced economy with only commodities in $M$ and preferences on this reduced commodity space obtained from the preferences in $\mathcal{E}$ with consumption of the commodities in $I \cup J$ equal to the initial endowments. At any $q^{0}$ inducing a trivial equilibrium we have that $r\left(q^{0}\right)=-w$, while at any $\bar{q}$ inducing an equilibrium with completely pessimistic expectations concerning demand opportunities of commodities in $I \cup J$ and Walrasian prices for the commodities in $M$, it holds that $r_{j}(\bar{q})=w_{j}$ for all $j \in I \cup J$, and $r_{j}(\bar{q})=0$ for all $j \in M$. Observe that $r_{j}\left(q^{0}\right)=-w_{j}$ for all $j \in I$ implies that for some $\mu^{0}>0$

$$
\sum_{j \in I} q_{j}^{0}=\sum_{j \in I}\left(\mu^{0} r_{j}\left(q^{0}\right)+p_{j}\left(q^{0}\right)\right)=-\mu^{0} \sum_{j \in I} w_{j}+\sum_{j \in I} p_{j}\left(q^{0}\right)=-\mu^{0} \sum_{j \in I} w_{j}+1<1
$$

and that $r_{j}(\bar{q})=w_{j}$ for all $j \in I$ implies that for some $\bar{\mu}>0$

$$
\sum_{j \in I} \bar{q}_{j}=\sum_{j \in I}\left(\bar{\mu} r_{j}(\bar{q})+p_{j}(\bar{q})\right)=\bar{\mu} \sum_{j \in I} w_{j}+\sum_{j \in I} p_{j}(\bar{q})=\bar{\mu} \sum_{j \in I} w_{j}+1>1 .
$$

From this we obtain the following corollary.

Corollary 5.4. Let $\mathcal{E}=\left(\left\{\mathcal{X}^{i}, \succcurlyeq^{i}, w^{i}\right\}_{i=1}^{m}, P\right)$ be an economy satisfying Assumptions $\mathrm{X}, \mathrm{U}$ and $\mathrm{W}$, and let the set $P$ be as specified in (6). Then there exists a connected set of QCEs, containing a fully supply-constrained no-trade equilibrium and the following types of equilibria:

(i) For any $j \in I \cup J$, an equilibrium induced by a point $q$ satisfying $r_{j}(q)=0$, i.e. a generalized Drèze equilibrium with respect to commodity $j \in I \cup J$;

(ii) An equilibrium induced by a point $q$ satisfying $r_{j}(q)=0$ for all $j \in M$, i.e. a generalized Drèze equilibrium without binding expected trade opportunities for commodities in $M$;

(iii) An equilibrium induced by a point $q$ satisfying $\max _{j \in I_{n}} r_{j}(q)=0$, i.e. a supply-constrained equilibrium, so without binding expected opportunities for at least one of the commodities;

(iv) An equilibrium induced by a point $q$ satisfying $\max _{j \in I \cup J} r_{j}(q)=0$, i.e. a supply-constrained equilibrium without binding expected trade opportunities for at least one of the commodities in $I \cup \mathrm{J}$;

(v) An equilibrium induced by a point $q$ satisfying $\sum_{j \in I} q_{j}=1$, i.e. for a commodity in I there may only be binding expected supply (demand) opportunities if its price is on its lower (upper) bound;

(vi) An equilibrium with completely pessimistic expectations concerning the demands of the commodities in I $\cup \mathrm{J}$ and Walrasian prices for the reduced economy with commodities in $M$.

At an equilibrium of type (iv) it may hold for some $j \in M$ that $r_{j}(q)<0$ and hence there are binding expected supply opportunities for some of the commodities in $M$. At such an equilibrium we have that for some $h \in I \cup J, r_{h}(q)=0$ and $p_{h}(q) \geq$ $\underline{p}_{h}>0$ and thus $p_{h}(q)\left(-w_{h}-r_{h}(q)\right)<0$. Now, suppose that $r_{j}(q)<0$ and thus $p_{j}(q)=\varepsilon$ for some $j \in M$. From the monotonicity of the preferences it then follows for sufficiently small $\varepsilon$ that $d_{j}^{i}(q)=w_{j}^{i}+\left(w_{j}-r_{j}(q)\right)>w_{j}^{i}$ for all $i \in I_{m}$, contradicting that $z_{j}(q)=0$. Hence we must have that $r_{j}(q)=0$ for all $j \in M$, implying that there are no binding expected trade opportunities for all commodities in $M$.

Corollary 5.5. Let $\mathcal{E}=\left(\left\{\mathcal{X}^{i}, \succcurlyeq^{i}, w^{i}\right\}_{i=1}^{m}, P\right)$ be an economy satisfying Assumptions $\mathrm{X}, \mathrm{U}$ and $\mathrm{W}$, and let the set $P$ be as specified in (6). Then for $\varepsilon$ sufficiently close to zero, there exists a supply-constrained equilibrium without binding expected trade opportunities 
for all commodities in $M$ and at least one of the commodities in $I \cup J$. Further, if $I \cup J=\emptyset$, then the equilibrium of type (vi) is a Walrasian equilibrium for the economy $\mathcal{E}$ with price set $P=\left\{p \in \mathbb{R}_{+}^{n} \mid p_{j} \geq \varepsilon, j \in I_{n}\right\}$. In this case there is a connected set of equilibria connecting a fully supply-constrained no-trade equilibrium with a Walrasian equilibrium.

\section{Acknowledgement}

The authors thank an anonymous referee for his helpful comments on an earlier draft.

\section{References}

Browder, F.E., 1960. On continuity of fixed points under deformation of continuous mappings. Summa Brasilia Mathematica 4, $183-191$.

Chetty, V.K., Nayak, P.R., 1978. Drèze equilibria for polyhedral and strictly convex price sets. Discussion paper. Indian Statistical Institute, New Delhi, India. Citanna, A., Crès, H., Drèze, J., Herings, P.J.J., Villanacci, A., 2001. Continua of underemployment equilibria reflecting coordination failures, also at Walrasian prices. Journal of Mathematical Economics 36, 169-200.

Dehez, P., Drèze, J.H., 1984. On supply-constrained equilibria. Journal of Economic Theory 33, 172-182.

Drèze, J.H., 1975. Existence of an exchange economy under price rigidities. International Economic Review 16, 310-320.

Drèze, J.H., 1997. Walras-Keynes equilibria, coordination and macroeconomics. European Economic Review 41, 1737-1762.

Drèze, J.H., 2001. On the macroeconomics of uncertainty and incomplete markets. Recherches Economiques de Louvain 67, 1-29.

Drèze, J.H., Gollier, C., 1993. Risk sharing on the labour market. European Economic Review 37, 1457-1482.

Herings, P.J.J., 1996a. Static and Dynamic Aspects of General Equilibrium Theory. Kluwer Academic Publishers, Dordrecht, The Netherlands.

Herings, P.J.J., 1996b. Equilibrium existence results for economies with price rigidities. Economic Theory 7, 63-80.

Herings, P.J.J., 1998. On the existence of a continuum of constrained equilibria. Journal of Mathematical Economics 30, $257-273$.

Herings, P.J.J., Polemarchakis, H.M., 2005. Pareto improving price regulation when the asset market is incomplete. Economic Theory 25, 135-154.

Herings, P.J.J., Talman, A.J.J., Yang, Z., 1996. The computation of a continuum of constrained equilibria. Mathematics of Operations Research 21, 675-696.

Kurz, M., 1982. Unemployment equilibria in an economy with linked prices. Journal of Economic Theory 26, 110-123.

Roberts, J., 1987a. An equilibrium model with involuntary unemployment at flexible, competitive prices and wages. American Economic Review 77, 856-874.

Roberts, J., 1987b. General equilibrium analysis of imperfect competition: an illustrative example. In: Feiwel, G. (Ed.), Arrow and the Ascent of Modern Economic Theory. MacMillan, London, pp. 415-438.

Roberts, J., 1989a. Equilibrium without market clearing. In: Cornet, B., Tulkens, H. (Eds.), Contributions to Operations Research and Economics: The Twentieth Anniversary of CORE. MIT Press, Cambridge, MA, pp. 145-158.

Roberts, J., 1989b. Involuntary unemployment and imperfect competition: a game-theoretic macromodel. In: In: Feiwel, G.R. (Ed.), The Economics of Imperfect Competition and Employment: Joan Robinson and Beyond. MacMillan, London, pp. 146-165.

van der Laan, G., 1980. Equilibrium under rigid prices with compensation for the consumers. International Economic Review 21, 53-73.

van der Laan, G., 1982. Simplicial approximation of unemployment equilibria. Journal of Mathematical Economics 9, 83-97.

van der Laan, G., 1984. Supply-constrained fixed price equilibria in monetary economies. Journal of Mathematical Economics 13, 171-187.

Weddepohl, C., 1987. Supply-constrained equilibria in economies with indexed prices. Journal of Economic Theory 43, 203-222. 